\title{
The impact of antimicrobial stewardship strategies on antibiotic appropriateness and prescribing behaviours in selected countries in the Middle East: a systematic review
}

Ziad Nasr, ${ }^{1}$ Bridget Paravattil ${ }^{7}$ and Kyle J. Wilby ${ }^{7}$

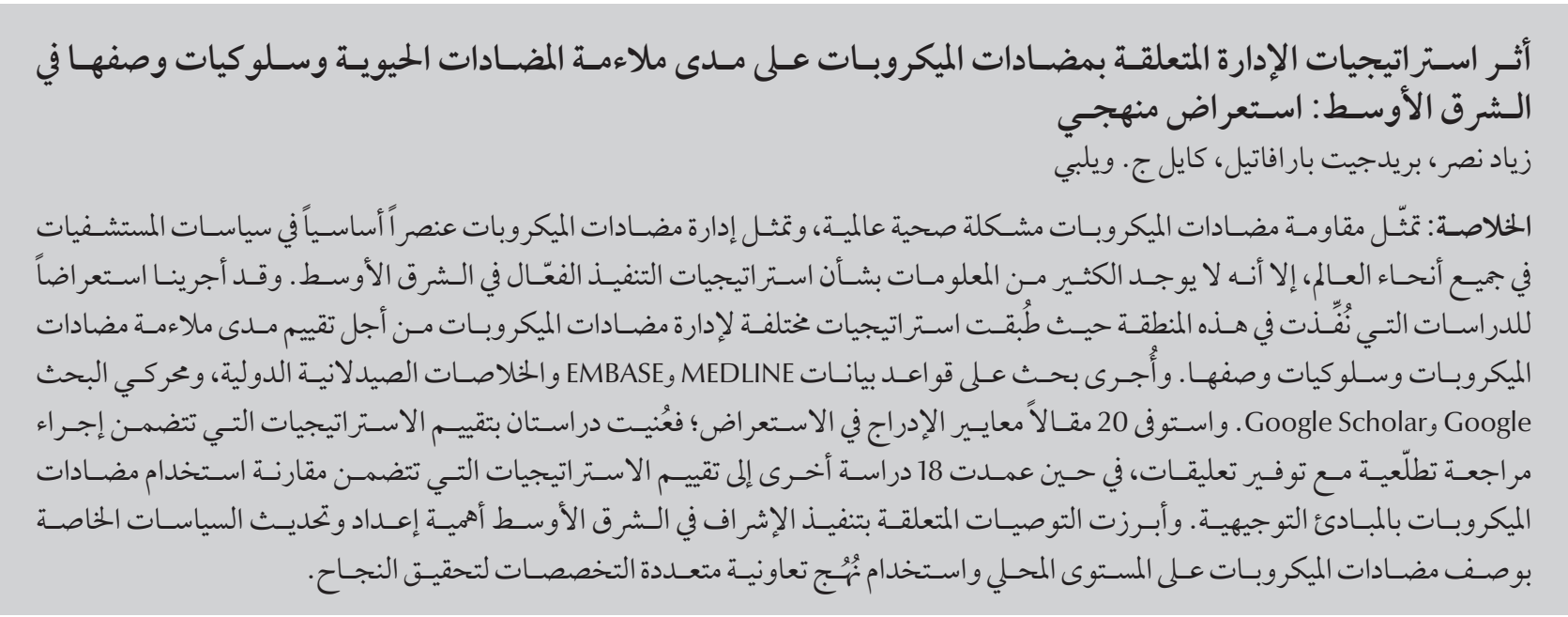

ABSTRACT Antimicrobial resistance is a global health problem and antimicrobial stewardship is an essential component of hospital policies worldwide yet little is known regarding effective implementation strategies in the Middle East. We conducted a review of studies carried out in this region that deployed different antimicrobial stewardship strategies to assess antimicrobial appropriateness and prescribing behaviours. A search of MEDLINE, EMBASE, International Pharmaceutical Abstracts, Google and Google Scholar was conducted. Twenty articles met the inclusion criteria; 2 studies evaluated strategies including prospective audit with feedback, while 18 others evaluated strategies including benchmarking antimicrobial utilization against guidelines. Recommendations for implementation of stewardship in the Middle East highlighted the importance of developing and updating local antimicrobial prescribing policies and using collaborative interdisciplinary approaches for success.

L'impact des stratégies de gestion des antimicrobiens sur la pertinence des antibiotiques et les comportements en matière de prescription au Moyen-Orient : analyse systématique

RÉSUMÉ La résistance aux antimicrobiens est un problème de santé mondial et la gestion des antimicrobiens constitue une composante essentielle des politiques hospitalières dans le monde. Pourtant, il existe actuellement peu d'informations sur l'efficacité des stratégies de mise en œuvre au Moyen-Orient. Nous avons passé en revue des études conduites dans la région utilisant différentes stratégies de gestion des antimicrobiens afin d'évaluer la pertinence des antimicrobiens et les comportements en matière de prescription. Une recherche dans Medline, Embase, International Pharmaceutical Abstracts, Google et Google Scholar a été effectuée. Vingt articles répondaient au critère d'inclusion, deux études évaluaient les stratégies incluant des audits prospectifs avec rétro-information, tandis que 18 autres évaluaient des stratégies incluant des analyses comparatives de I'utilisation des antimicrobiens par rapport aux procédures. Les recommandations en matière de mise en œuvre de la gestion des antimicrobiens au Moyen-Orient soulignaient l'importance de développer et de mettre à jour les politiques locales de prescription des antimicrobiens, et de recourir à des approches interdisciplinaires collaboratives, si l'on veut assurer le succès des programmes. 


\section{Introduction}

Antimicrobial resistance is known to be one of the major threats to global health, mainly due to the prevalence of injudicious and overzealous use of antimicrobials (1). The world has reached a post-antibiotic era where major and even minor injuries can lead to multidrug-resistant infections and result in mortality, as available antibiotic options may not be available for treatment (1). This is alarming, especially that resistant bacterial illnesses increases the cost of treatment and extends the course of therapy. As such, the duration of hospitalizations, the overall health care costs and the economic burden on families and societies worldwide will increase (1). In fact, the World Health Organization (WHO) states that multidrugresistant bacterial infections have led to more than 8 million additional hospital stays and currently cost the health care system in excess of US $\$ 20$ billion (2).

Antimicrobial stewardship refers to a set of coordinated strategies that focus on promoting appropriate antibiotic use in inpatient health care settings while improving patient outcomes, ensuring patient safety, reducing pharmacy cost for antibiotics and decreasing antimicrobial resistance and the spread of infections caused by multidrug-resistant organisms $(3,4)$. Antimicrobial stewardship programmes are typically hospital-based programmes designed to ensure that patients receive the right antibiotic, at the right dose, at the right time and for the right duration (5-7). An effective programme is one that has committed leadership and necessary human, financial and information technology resources. The literature shows that successful programmes are those that are led by a coalition between physicians and clinical pharmacists $(8-10)$. While most research conducted is reported from centres in North America and Europe, international guidelines are currently being developed and disseminated. However, institutions in the
Middle East and the Persian Gulf region still lack firm and clear guidelines for proper antimicrobials use, which are essential for the success of local programmes due to regional variations in antibiotic utilization and prevalence of resistant organisms (10).

Antimicrobial stewardship interventions can only be successful if they meet the specific needs of the health care institution with dedicated multifaceted, multidisciplinary teams (physicians, pharmacists and nurses), administrators and policy-makers (9). These interventions include: prospective audits with intervention and feedback; implementation of formulary restriction programmes and pre-authorization requirements for specific antimicrobials within individual institutions; education; compliance with local/national guidelines and dissemination of clinical pathways; antimicrobial cycling and order forms; streamlining or therapy de-escalation; use of combination antimicrobial therapy; and dose optimization, or the switch from the intravenous route of administration to oral when indicated (7). In general, such interventions typically require essential components such as stakeholder buy-in, review of documentation systems, navigation of prescriber-pharmacist relationships and national/institutional prescribing policies $(11,12)$.

In order to address the unique needs of individual institutions through implementation of a proactive antimicrobial stewardship programme and to provide guidance for future programme development, we conducted a review of studies that adopted different antimicrobial stewardship strategies in the Middle East region to assess antimicrobial utilization and evaluate antimicrobial prescribing behaviours within hospitals. A secondary objective was to determine core recommendations for programme development in the Middle Eastern context.

\section{Methods}

A search of MEDLINE (1948-February 2016), EMBASE (1980February 2016), International Pharmaceutical Abstracts (1970February 2016), Google and Google Scholar was conducted for articles assessing prescribing patterns of antibiotics and evaluating the use of antimicrobials within health care institutions in selected countries in the Middle East region. Search terms included combinations of 'antimicrobial', 'antimicrobial stewardship', 'prescribing, 'utilization', 'resistance', and 'Middle East', 'Bahrain', 'Iraq', 'Jordan', 'Kuwait', 'Lebanon', 'Oman', 'Palestine', 'Qatar', 'Saudi Arabia, 'Syria', 'United Arab Emirates' and 'Yemen'. The reference lists of the articles identified were manually searched for pertinent articles that were not identified in the electronic search. Identified abstracts were included if the study was published in English and had at least 1 antibiotic prescribing pattern or its use was assessed or evaluated in a hospital inpatient setting. All populations were included. Articles were excluded if they were done in a community setting (including health centres or primary health clinics), addressed general drug prescribing patterns with no focus on antibiotics or if they did not assess use against guidelines or defined local institutional or national policies.

After title and abstract review, the full text versions of identified articles were downloaded for review. After assessment against inclusion criteria, a total of 20 articles were included in this narrative review. Data extracted from identified studies included study design, setting, interventions, comparators and results. If provided by the article, recommendations regarding programme development or implementation were also extracted. 


\section{Results}

\section{Strategies for implementation of successful antimicrobial stewardship programmes}

All 20 studies collected data through patients' chart s and medical file review, either retrospectively (11 studies) or prospectively (9 studies). All studies provided recommendations for successful programme development and/ orimplementation. Table 1 summarizes the studies in regard to the adoption by institutions of interventional strategies in order to assess and enhance antimicrobial use and prescribing behaviours by physicians (13-32).

Two studies used proactive core strategies in the form of prospective audit or point prevalence survey followed by education and feedback $(13,14)$ to evaluate the appropriateness of antimicrobial prescribing and utilization. Amer et al. compared the prescribing appropriateness of the empiric antibiotic therapy before and after the implementation of the antimicrobial stewardship programme at their institution in Saudi Arabia (13). The primary aim of the programme was to optimize the appropriateness of antimicrobials use and thus prevent the emergence of antimicrobial resistance associated with inappropriate use. Through targeting the 5 most commonly used broad-spectrum antibiotics (piperacillin/tazobactam, imipenem/cilastatin, meropenem, vancomycin, tigecycline) in the intensive care unit setting, they prospectively compared patients who were put under the active antimicrobial stewardship programme arm with other patients who had been admitted to the same unit at that institution before the implementation of the programme in 2011 over a 6-month period during the same season. Appropriateness of empirical antibiotics was assessed by the stewardship team and evaluated according to the institution's internally developed criteria, including formulary restrictions, and following reliable international infectious diseases clinical guidelines. Recommendations were then communicated in a verbal fashion to the intensive care unit team. The appropriateness of empirical antibiotics improved from $30.6 \%(15 / 49)$ in the historical control arm to $100 \%(24 / 24)$ in the proactive antimicrobial stewardship programme arm; the difference was statistically significant $(P<0.05)$. The rate of inappropriate antimicrobial use was reduced to $0 \%$ upon implementing the programme. There was also a significant reduction in the antibiotic utilization and direct costs in the antimicrobial stewardship programme arm. On the other hand, there was no significant clinical difference between the 2 arms of the study in terms of Clostridium difficile infection rate. A total of 27 interventions were made with an acceptance rate of $96.3 \%$ (13). However, this study had a number of limitations, including lack of randomization, small sample size, short follow-up duration and single institution's experience, thus affecting the generalizability of the results.

After performing a point prevalence survey over a 3-month period to assess vancomycin prescribing compliance with the Centers for Disease Control and Prevention (CDC) guidelines, Dib et al. evaluated feedback and performed an educational intervention to enhance adherence to local policies at their institution in Saudi Arabia (14). In the pre-intervention period, vancomycin utilization was deemed appropriate in only $65 \%(48 / 74)$ of adult patients. The investigators then implemented a continuous education and active feedback approach targeting compliance with CDC recommendations. During the post-intervention period, compliance with guidelines for vancomycin utilization increased significantly for all indicators from $65 \%(48 / 74)$ in the preintervention phase to $91 \%(31 / 34)$ in the post-intervention phase $(P=0.004)$. In addition, compliance with vancomycin trough level monitoring increased from $35 \%$ to $67.7 \%(P=0.0002)$. The study concluded that audit and feedback and/or educational approaches are effective in improving compliance with guidelines for antimicrobials utilization (14).

Eighteen additional studies evaluated prescribing patterns and antimicrobial utilization within hospitals in the Middle East area (15-32) by assessing compliance with local/national institutional policies or internationally endorsed clinical guidelines. Eleven (61.1\%) of these reported unwarranted and inappropriate use (typically overuse) of antimicrobials. The majority of the studies concluded that prescribing habits were to blame for the discrepancies observed, and that physicians were not prescribing according to local policies and guidelines (16-26). Conversely, 6 (33.3\%) studies reported adherence to guidelines and local institutional policies (27-32). These studies deemed prescribing patterns to be rational and in line with local and international standards. The reasons behind this are recorded as being the result of institutional policies, implementation of protocols or prescribing aids, or adoption of international guidelines from North America and/or Europe. Some of these studies also reported that antimicrobial stewardship initiatives (restrictive measures, antimicrobial cycling, antimicrobial switch, etc.) likely influenced adherence rates $(15,24,32)$.

\section{Recommendations for programme development and implementation}

Specific recommendations extracted from each study are given in Table 1 . All the studies we examined provided recommendations that are considered to be crucial for further enhancement of antimicrobial usage through implementation of successful antimicrobial stewardship programmes. Eight studies mentioned the importance of implementing local guidelines and policies $(13,16,18,21,24,25-27)$. It was also suggested that these should be supported 
by an infection control committee $(17,23)$. Other studies recommended that these policies should be updated regularly and must be readily available and disseminated throughout the institution $(17,20,23,24,26)$.

Other approaches were also mentioned. These included annual surveillance; infectious diseases team consultations $(17,23,24,32)$ focusing on interdisciplinary collaboration; and good cross-departmental communication $(22,23)$. In the majority of studies training of health care professionals, public health awareness and continuing medical education were considered to be important components that further enhance health care professionals' knowledge, and thus improve antibiotics use and overall prescribing appropriateness (13-15,19,21,24-26). Others highlighted the importance of the role of the clinical pharmacist in monitoring prescriptions (i.e. dosage and frequency adjustments), intervening when appropriate, and the implementation of such interventions if deemed reasonable $(17,18,21,23,29,30,32)$. Lastly, some studies reflected upon the need for continuous use of guidelines $(13,28,31)$ and the increased need for drug utilization reviews, audits, or strategies that include restriction policies, order forms, pharmacodynamics dose optimization and antimicrobial cycling and rotating $(13-15,17,21,23,24)$.

\section{Discussion}

To our knowledge, this is the first review to summarize published antimicrobial stewardship interventions in the Middle Eastern context and to extract recommendations from past studies for future programme implementation. The results provide excellent background information for institutions in the region attempting to improve antimicrobial use and an insight for programme development and implementation. A total of 20 studies were identified that have assessed at least one type of antimicrobial stewardship intervention. Two of these studies reported the use of proactive core interventions which positively affected prescribing behaviours through audit and feedback. The remaining 18 papers primarily described adherence of antimicrobial prescribing patterns to institutional, local/national, or international policies and guidelines. The end result from the studies we examined in this review was that antimicrobial utilization is a problem in the Middle East and more-proactive strategies should be developed in order to further improve the appropriateness of therapy.

The 2 papers describing proactive core strategies must be closely examined to determine how results can be applied in other settings in the region $(13,14)$. These studies are very important, in that they demonstrate that a prospective audit and feedback approach is effective in the region, and their findings suggest that prescribers were receptive and an audit and feedback model can be established. While this approach is a core component of antimicrobial stewardship programmes in North America and Europe, there may be cultural considerations in the Middle East (physician attitudes, acceptance of collaborative practices, acceptance of pharmacist recommendations, etc.) that could limit effectiveness $(8,33)$. These cultural differences should be further explored and any associated impact on effectiveness of programmes should be assessed. In addition, a key finding was that education seems to be central in the implementation of any programme and perhaps is important for those institutions developing programmes in areas of the Middle East where antimicrobial stewardship is considered a novel concept (34).

A number of key recommendations were extracted from the studies included in our review that provide insight into future programme development and implementation; many themes emerged from these recommendations that warrant discussion. First, development of local policies was recommended by a large majority of the studies. The importance of this point cannot be stressed enough as antimicrobial resistance patterns vary greatly between settings and even between institutions. Therefore, antibiograms and prescribing protocols and policies are essential for continuous monitoring of outcomes at the institutional level $(11,35)$. It is also important to note that any guideline established from other settings (i.e. North America or Europe) must be carefully reviewed for applicability before adoption in a new context.

Secondly, any policy or prescribing protocol developed must be continually updated based on current epidemiological data, including infection prevalence and antimicrobial resistance. This should be done at least annually in order to ensure guidelines and recommendations remain valid over time.

A third point that emerged from the recommendations was the importance of the collaborative approach within a multidisciplinary team. Physicians and clinical pharmacists are typically considered to be the core members of antimicrobial stewardship programme leadership (in addition to information technology personnel), however collaboration with other disciplines such as nursing, laboratory personnel, administrators and home care services will only improve stakeholder buy in and promote a culture of antimicrobial stewardship within the institution itself $(9,10,36)$.

Considering the points discussed above, we urge institutions in the Middle East to consider the following points for programme development and implementation. First, it is important to ensure adequate baseline surveillance data is obtained, in order to guide the most appropriate type of interventions and stewardship strategies. This can be obtained through laboratory data, drug utilization trends and point prevalence surveys. In addition, it is important for 


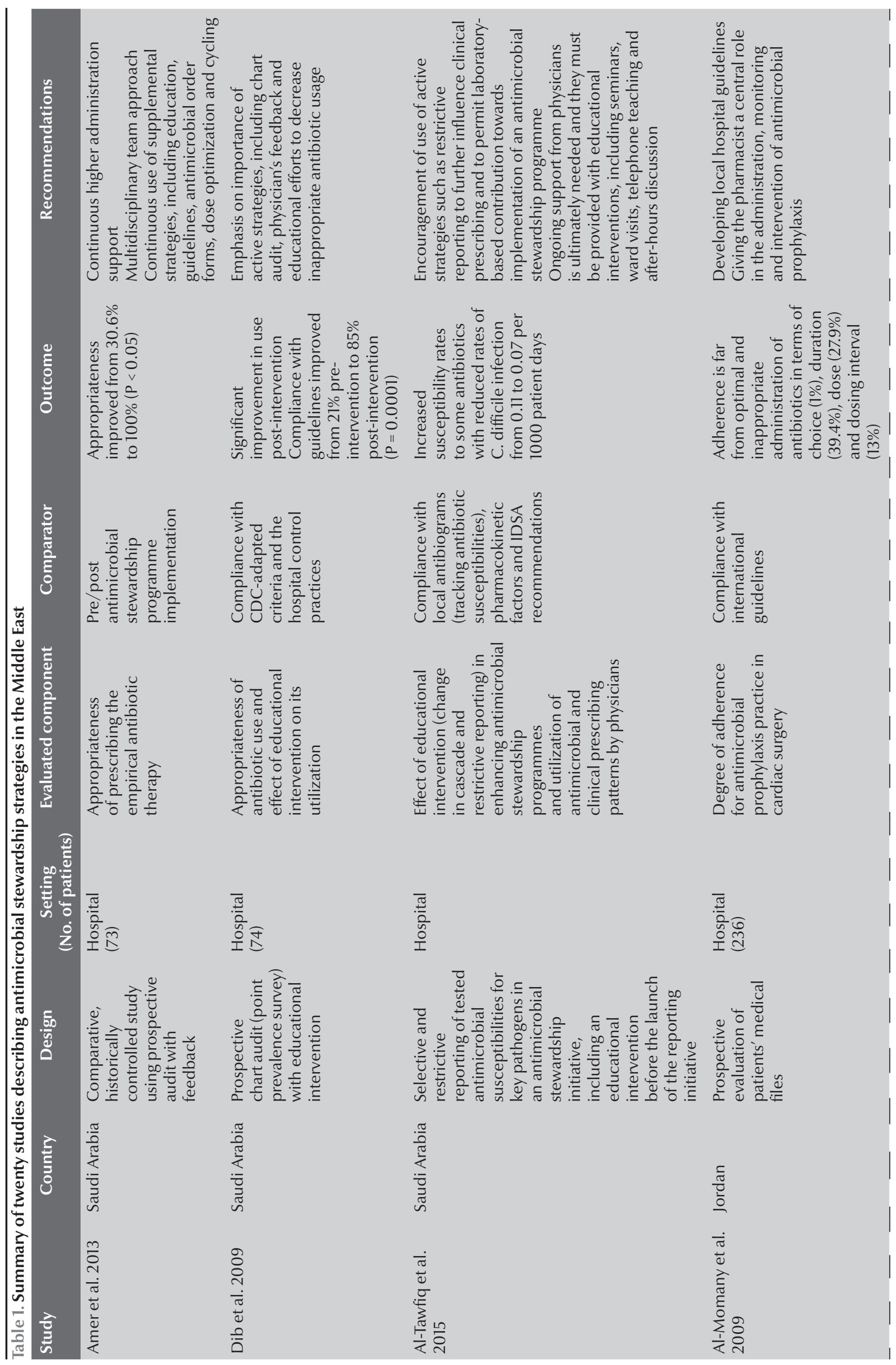




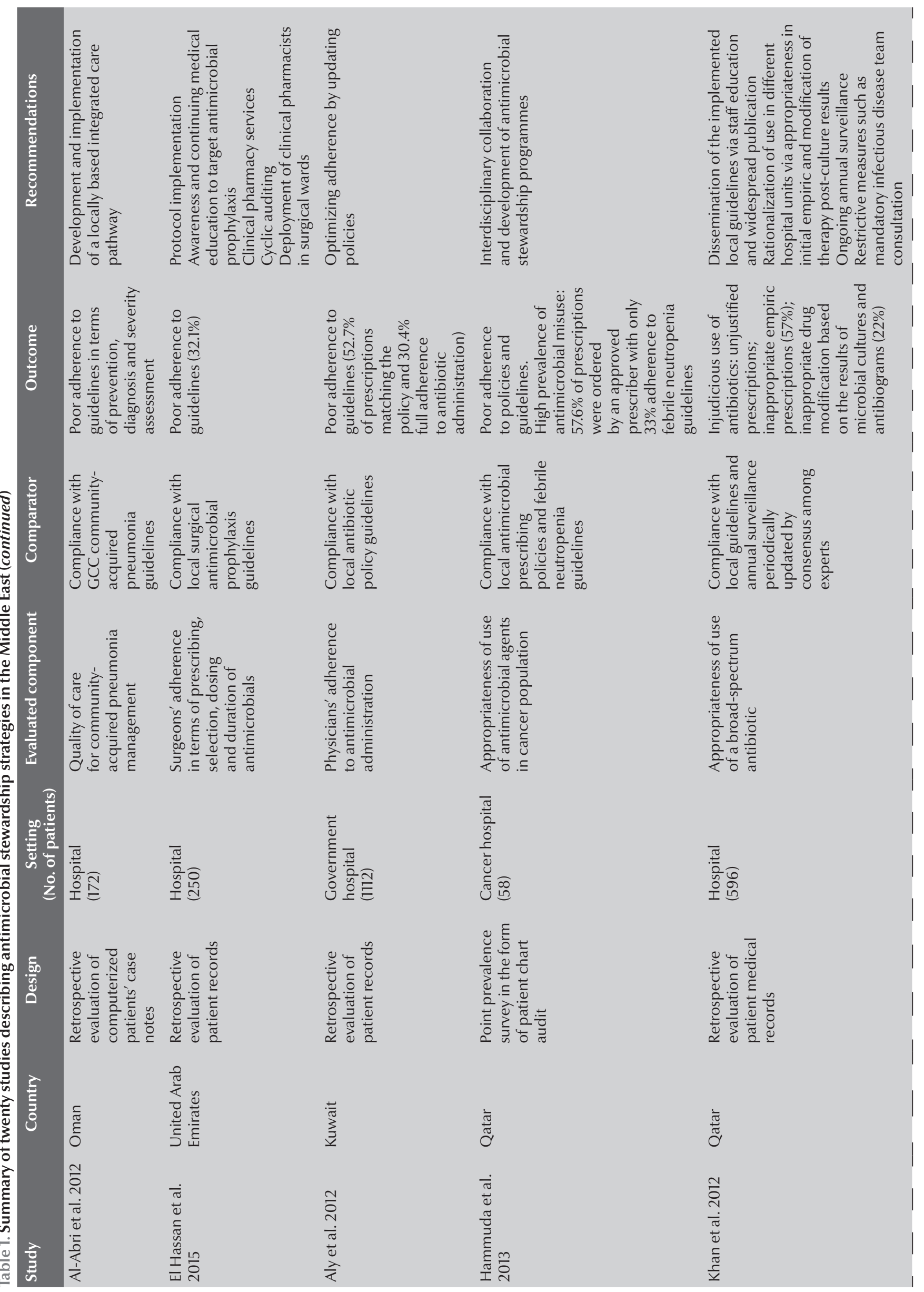




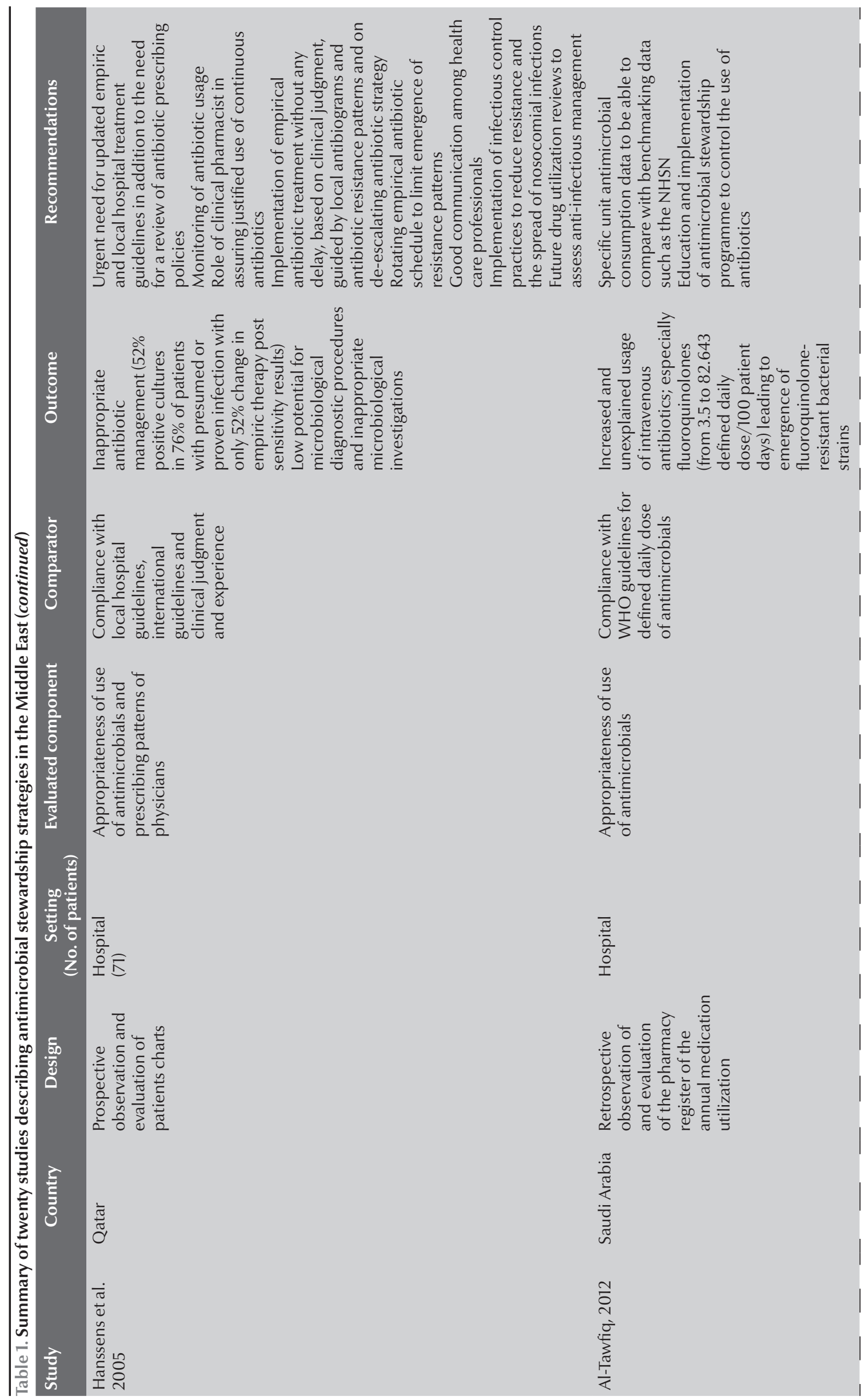




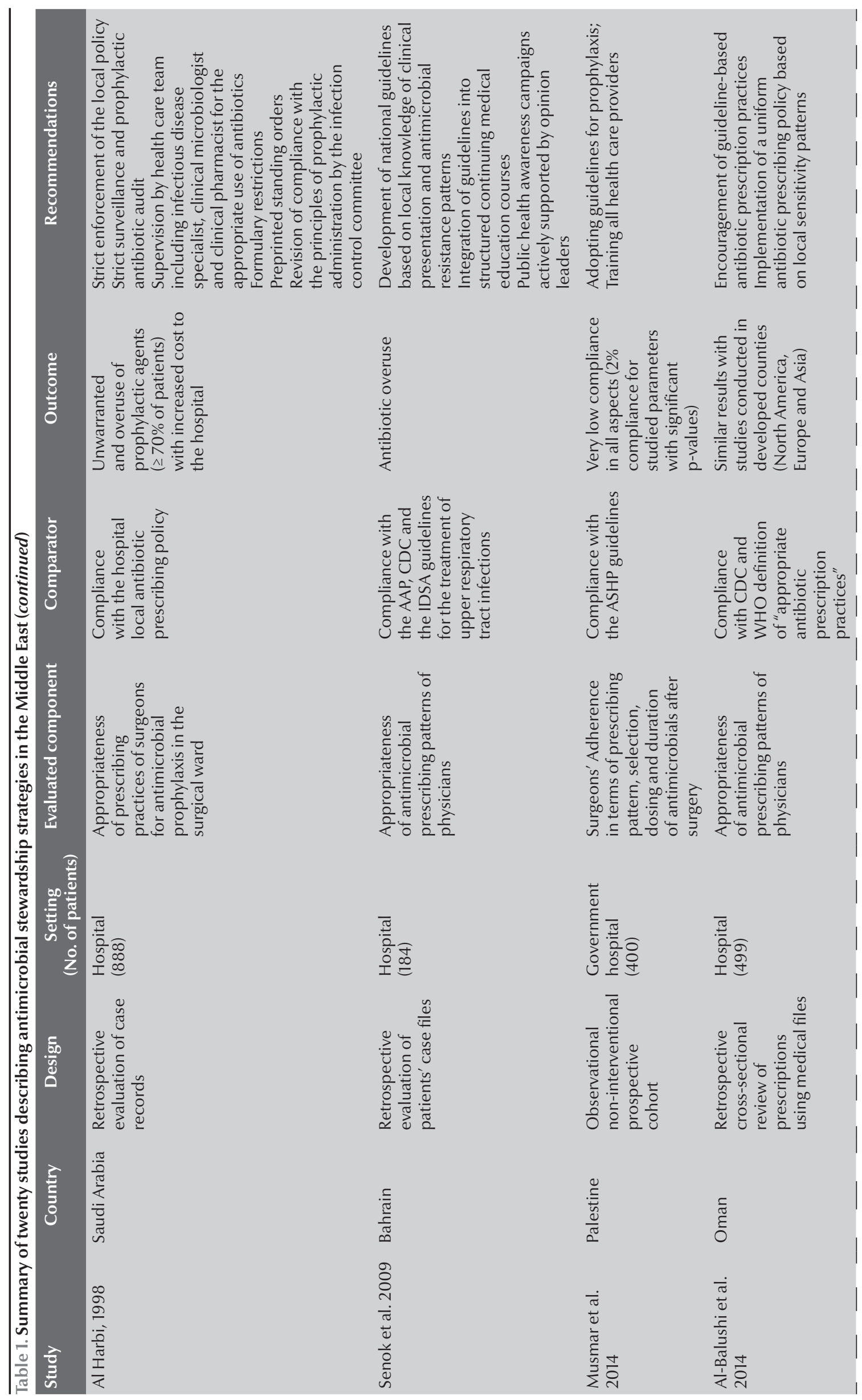




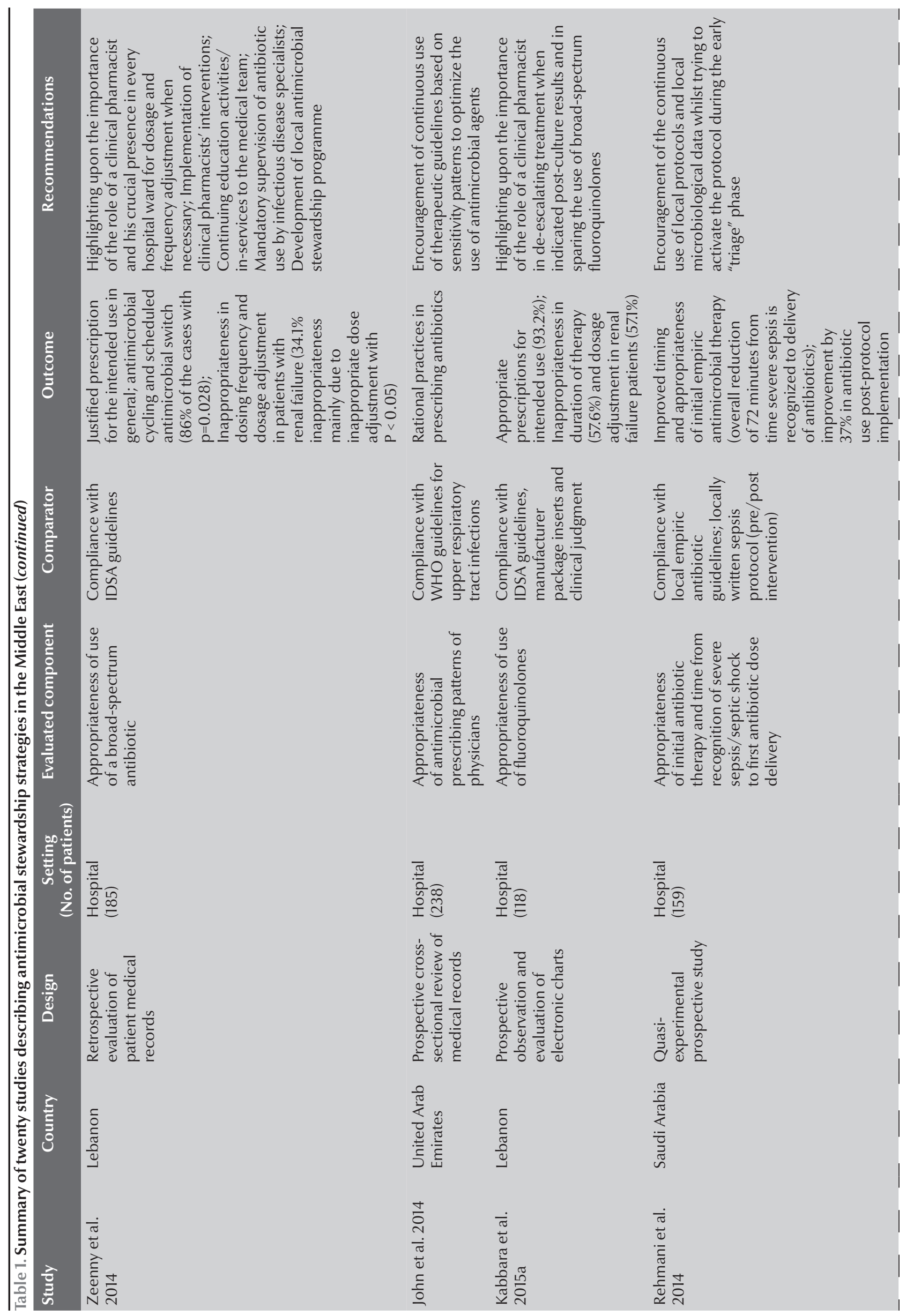




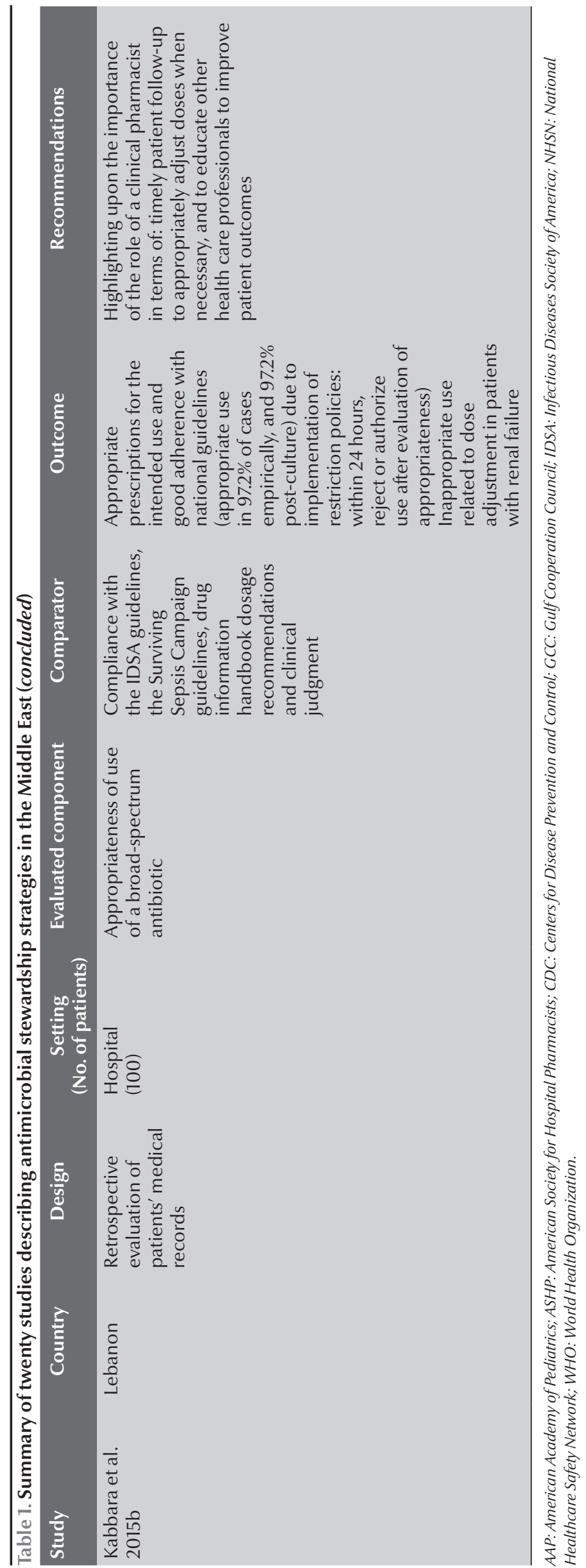

institutions to evaluate any antimicrobial stewardship initiative on a regular basis (e.g. annually) in order to assess the impact and seek opportunities for improvement. Second, any adapted policy or guideline originating from another context must be locally validated to ensure appropriateness and applicability for the setting. While international guidelines do provide evidence-based recommendations, stewardship must be adapted to each local context and culture. Lastly, we strongly urge clinicians and researchers working in this area to disseminate their findings in the form of abstracts and publications. As demonstrated by this review, there is a great lack of data for guidance of stewardship in the Middle Eastern context, and sharing of experiences and knowledge will only work towards improving patient outcomes through appropriate use of antimicrobials.

\section{Conclusions}

Our findings show antimicrobial stewardship programmes are in their infancy in the region but that work is currently being done to further develop these programmes. Our findings also show that antimicrobial utilization is suboptimal in the region, as demonstrated by the studies we surveyed. We urge those working in Middle Eastern contexts to make antimicrobial stewardship an institutional priority and to consider recommendations identified by other centres in the region when developing and implementing new strategies. By doing so, we can work towards improving the use of antimicrobials in the region and ultimately bettering patient health outcomes.

Funding: None.

Competing interests: None declared. 
1. Antimicrobial resistance: global report on surveillance 2014. Geneva: World Health Organization; 2014 (http://www.who. int/drugresistance/documents/surveillancereport/en/, accessed 20 March 2017).

2. Leung E, Weil DE, Raviglione M, Nakatani H. The WHO policy package to combat antimicrobial resistance. Bull World Health Organ. 2011;89:390-2. PMID: 21556308

3. Doron S, Davidson LE. Antimicrobial stewardship. Mayo Clin Proc. 2011;86(11):1113-23. PMID:22033257

4. Goff DA, Rybak MJ. Introduction to the special issue on antimicrobial stewardship. Pharmacotherapy. 2012;23(8):663-4. PMID:23307515

5. Dellit TH, Owens RC, McGowan JE Jr, Gerding DN, Weinstein RA, Burke JP, et al. Infectious Diseases Society of America and the Society for Healthcare Epidemiology of America guidelines for developing an institutional program to enhance antimicrobial stewardship. Clin Infect Dis. 2007;44(2):159-77. PMID:17173212

6. Paterson DL. The role of antimicrobial management programs in optimizing antibiotic prescribing within hospitals. Clin Infect Dis. 2006; 42:S90-5. PMID:16355322

7. Tamma PD, Cosgrove SE. Antimicrobial stewardship. Infect Dis Clin North Am. 2011;25(1):245-60. PMID:21316003

8. Bal AM, Gould IM. Antibiotic stewardship: overcoming implementation barriers. Curr Opin Infec Dis. 2011;24(4):357-62. PMID:21587070

9. Drew RH. Antimicrobial stewardship programs: how to start and steer a successful program. J Manag Care Pharm. 2009:15:S18-23. PMID:19236137

10. Khalili H, Farsaei S, Rezaee H, Dashti-Khavidaki S. Role of clinical pharmacists' interventions in detection and prevention of medication errors in a medical ward. Int J Clin Pharm. 2011;33(2):281-4. PMID:21394569

11. Goldmann DA, Weinstein RA, Wenzel RP, Tablan OC, Duma RJ, Gaynes RP, et al. Strategies to prevent and control the emergence and spread of antimicrobial-resistant microorganisms in hospitals: a challenge to hospital leadership. JAMA. 1996;275(3):234-40. PMID:8604178

12. Yates RR. New intervention strategies for reducing antibiotic resistance. CHEST. 1999;115:24S-27S. PMID:10084456

13. Amer MR, Akhras NS, Mahmood WA, Al-Jazairi AS. Antimicrobial stewardship program implementation in a medical intensive care unit at a tertiary care hospital in Saudi Arabia. Ann Saudi Med. 2013;33(6):547-54. PMID:24413857

14. Dib JG, Al-Tawfiq JA, Al Abdulmohsin S, Mohammed K, Jenden PD. Improvement in vancomycin utilization in adults in a Saudi Arabian medical center using the Hospital Infection Control Practices Advisory Committee guidelines and simple educational activity. J Infect Public Health. 2009;2(3):141-6. PMID:20701874

15. Al-Tawfiq JA, Momattin H, Al-Habboubi F, Dancer SJ. Restrictive reporting of selected antimicrobial susceptibilities influences clinical prescribing. J Infect Public Health. 2015;8(3):234-41. PMID:25466592

16. Al-Abri SS, Al-Maashani S, Memish ZA, Beeching NJ. An audit on inpatient management of community-acquired pneumonia in Oman: a comparison with regional clinical guidelines. J Infect Public Health. 2012;5(3):250-6. PMID:22632599

17. Al-Harbi M. Antimicrobial prophylactic practice in surgical patients. East Afr Med J. 1998;75(12):703-7. PMID:10065210

18. Al-Momany NH, Al-Bakri AG, Makahleh ZM, Wazaify MM. Adherence to international antimicrobial prophylaxis guidelines in cardiac surgery: A Jordanian study demonstrates need for quality improvement. J Manag Care Pharm. 2009;15(3):262-71. PMID:19326957

19. Al-Tawfiq JA. Changes in the pattern of hospital intravenous antimicrobial use in Saudi Arabia, 2006-2008. Ann Saudi Med. 2012;32(5):517-20. PMID:22871622
20. Aly NY, Omar AA, Badawy DA, Al-Mousa HH, Sadek AA. Audit of physician's adherence to the antibiotic policy guidelines in Kuwait. Med Princ Pract. 2012;21:310-17. PMID:22236835

21. El-Hassan M, Elnour AA, Farah HH, Shehab A, Al Kalbani NM, Asim S, et al. Clinical pharmacists' review of surgical antimicrobial prophylaxis in a tertiary hospital in Abu Dhabi. Int J Clin Pharm. 2015;37(1):18-22. PMID:25488316

22. Hammuda A, Hayder S, Elazzazy S, Black E. Point prevalence survey of antimicrobial utilization in oncology patients. J Infect Dev Ctries. 2013;7(12):990-3. PMID:24334947

23. Hanssens Y, Ismaeil BB, Kamha AA, Elshafie SS, Adheir FS, Saleh TM, et al. Antibiotic prescribing in a medical intensive care unit in Qatar. Saudi Med J. 2005;26(8):1269-76. PMID:16127527

24. Khan FY, Elhiday A, Khudair IF, Yousef H, Omran AH, Alsamman $\mathrm{SH}$, et al. Evaluation of the use of piperacillin/tazobactam $\left(\right.$ Tazocin $\left.^{\circledR}\right)$ at Hamad General Hospital, Qatar: are there unjustified prescriptions? Infect Drug Resist. 2012;5:17-21. PMID:22294859

25. Musmar SM, Ba 'ba H, Owais A. Adherence to guidelines of antibiotic prophylactic use in surgery: a prospective cohort study in North West Bank, Palestine. BMC Surg. 2014;14:69. PMID:25204205

26. Senok AC, Ismaeel AY, Al-Qashar FA, Agab W. Pattern of upper respiratory tract infections and physicians' antibiotic prescribing practices in Bahrain. Med Princ Pract. 2009;18(3):170-4 PMID:19349717

27. Al-Balushi K, Al-Ghafri F, Al-Sawafi F, Al-Zakwani I. Antibiotic prescribing trends in an Omani paediatric population. Sultan Qaboos Univ Med J. 2014;14(4):e495-9. PMID:25364552

28. John LJ, Cherian M, Sreedharan J, Cherian T. Patterns of antimicrobial therapy in acute tonsillitis: A cross-sectional hospitalbased study from UAE. An Acad Bras Cienc. 2014;86(1):451-7. PMID:24676179

29. Kabbara WK, Ramadan WH, Rahbany P, Al-Natour S. Evaluation of the appropriate use of commonly prescribed fluoroquinolones and the risk of dyslycemia. Ther Clin Risk Manag. 2015;11:639-47. PMID:25960658

30. Kabbara WK, Nawas GT, Ramadan WH. Evaluation of the appropriateness of imipenem/cilastatin prescription and dosing in a tertiary care hospital. Infect Drug Resist. 2015;8:31-8. PMID:25960658

31. Rehmani RS, Memon JI, Al-Gammal A. Implementing a collaborative sepsis protocol on the time to antibiotics in an emergency department of a Saudi hospital: Quasi randomized study. Crit Care Res Pract. 2014:410430. PMID:24818017

32. Zeenny R., Nasr Z, Adaimy I. Retrospective evaluation of the appropriate use of piperacillin/tazobactam in a tertiary care teaching hospital in Lebanon. Acta Medica Mediterranea. 2014;30:655-63.

33. Howard P, Pulcini C, Levy Hara G, West RM, Gould IM, Harbarth $\mathrm{S}$, et al. An international cross-sectional survey of antimicrobial stewardship programmes in hospitals. J Antimicrob Chemother. 2015;70(4):1245-55. PMID:25527272

34. Fishman NO. Antimicrobial stewardship. Am J Infect Control. 2006;S55-63. PMID:16813983

35. Ramsamy Y, Muckart DJ, Han KS. Microbiological surveillance and antimicrobial stewardship minimize the need for ultrabroad-spectrum combination therapy for treatment of nosocomial infections in a trauma intensive care unit: an audit of an evidence-based empiric antimicrobial policy. S Afr Med J. 2013;103(6):371-6. PMID:23725954

36. Boyles TH, Whitelaw A, Bamford C, Moodley M, Bonorchis K, Morris V, et al. Antimicrobial stewardship ward rounds and a dedicated chart reduce antibiotic consumption and pharmacy costs without affecting inpatient mortality or re-admission rates. PLoS One. 2013;8(12):e7974. PMID:24348995 